\title{
Resposta hematológica do cascudo ornamental amazônico Peckoltia oligospila ao estresse de transporte
}

[Hematological response of Amazonian ornamental Peckoltia oligospila subjected to stress by transportation]

\author{
M.S. Neves ${ }^{1}$, M.V.S. Couto ${ }^{1}$, N.C. Sousa ${ }^{1}$, R.F.B. Santos ${ }^{2}$, H.M. Dias ${ }^{3}$, H.A. Abe ${ }^{1}$, \\ J.A.R. Dias $^{1}$, F.S. Cunha ${ }^{4}$, M. Tavares Dias ${ }^{5}$, R.Y. Fujimoto ${ }^{6 *}$
}

\author{
${ }^{1}$ Aluno de pós-graduação - Universidade Federal do Pará - Castanhal, PA \\ ${ }^{2}$ Aluno de pós-graduação - Universidade Federal de Pernambuco - Recife, PE \\ ${ }^{3}$ Aluno de pós-graduação - Universidade Nilton Lins - Manaus - AM \\ ${ }^{4}$ Aluno de pós-graduação - Universidade Tiradentes - Aracaju - SE \\ ${ }^{5}$ Embrapa Amapá - Macapá, AP \\ ${ }^{6}$ Embrapa - Tabuleiros Costeiros - Aracaju, SE
}

\begin{abstract}
RESUMO
O objetivo deste trabalho foi avaliar as respostas hematológicas do acari-bola Peckoltia oligospila submetido ao estresse de transporte. Variações nos parâmetros de sangue foram analisadas às zero, seis, 24, 48, 72 e 96 horas após o transporte. Respostas ao estresse foram observadas entre zero e seis horas do transporte, mas a maioria dos parâmetros retornou aos valores basais em 24 horas. O tempo de zero hora (momento imediato após transporte) foi o mais crítico, com valores elevados de glicemia, eritrócitos e eritroblastos. Respostas secundárias tardias foram observadas para a proteína plasmática total, o volume corpuscular médio (VCM) e a hemoglobina corpuscular média (HCM) em seis horas após o transporte dos peixes, retornando aos valores basais após esse período. O número de leucócitos não sofreu alterações após o transporte. $\mathrm{O}$ estresse de transporte não comprometeu a fisiologia de $P$. oligospila, o que indica que esse peixe é resistente ao estresse se comparado com outras espécies. Porém, recomenda-se que não se realize qualquer outro procedimento estressante durante pelo menos 24 horas da recuperação dos peixes após transporte, para garantir a saúde e a sobrevivência dos animais transportados.
\end{abstract}

Palavras-chave: peixe endêmico, fisiologia, sangue, sanidade

\begin{abstract}
The objective of this work was to evaluate the hematological responses of bola-pleco (Peckoltia oligospila) undergoing the stress of transportation. Variations on blood parameters were analyzed at 0 , 6, 24, 48, 72 and $96 \mathrm{~h}$ after transportation. Responses to stress were detected from 0 to $6 \mathrm{~h}$ after the transportation of fish, however, most parameters returned to baselines values within $24 \mathrm{~h}$ of transportation. The moment of Oh was the most critical, presenting higher values of glycemia, erythroblasts and erythrocytes. Late secondary responses were observed to total plasmatic protein, mean corpuscular volume (MCV) and mean corpuscular hemoglobin (MCH) at 6h, returning to baselines values after this time. Leukocyte number was not affected by stress of transportation. The stress by transportation was not severe to influence the health of P. oligospila, indicating that fish is resistant to stress if compared to other species. However, we recommended no stressful procedures for at least 24 hours for recovery, in order to ensure health and survival of fish.
\end{abstract}

Keywords: endemic fish, physiology, blood, health

\section{INTRODUÇÃO}

O transporte inadequado pode comprometer a comercialização de peixes ornamentais, pois

Recebido em 26 de setembro de 2016

Aceito em 3 de dezembro de 2016

*Autor para correspondência (corresponding author)

E-mail: ryfujim@hotmail.com nesse procedimento estímulos estressores, tais como a captura, a alta densidade de estocagem e a má qualidade de água desafiam a homeostase dos peixes (Adamante et al., 2008; Oliveira et al., 2009; Lemos et al., 2015). A quebra da 
homeostase pode ser notada após alguns dias do evento indutor do estresse, quando ocorre uma diminuição na alimentação e o aparecimento de infecções secundárias nos peixes estressados (Harper, 2003; El-Galil e Aboelhadid, 2012). Esse conjunto de fatores torna o transporte um aspecto limitante na cadeia produtiva de peixes ornamentais, que pode diminuir a produção e a produtividade dessa atividade.

Apesar da existência de estudos relacionados às repostas dos peixes ao estresse causado pelo transporte (Adamante et al., 2008; Oliveira et al., 2009; Braun e Nuñes, 2014; Souza Neves et al., 2014), há falta de estudos para os peixes ornamentais. Entre esses peixes, estão aqueles da família Loricariidae, que possuem alto valor de mercado, pois são exportados principalmente para a Europa e os Estados Unidos. Portanto, são submetidos a longos tempos de transporte até o país de destino (Chao, 2001; Ribeiro et al., 2009). Entre esses Loricariidae, o acari-bola Peckoltia oligospila Günther, (1864), destaca-se por ser uma espécie endêmica da bacia hidrográfica do Rio Guamá, nordeste do estado do Pará. Na região, esse peixe representa $56 \%$ da receita gerada pela pesca ornamental (Prang, 2007; Torres, 2007). Além de seu elevado valor de mercado para exportação, o local de captura desse peixe é estratégico devido à proximidade com a capital do estado, Belém, de onde os peixes são exportados (Torres, 2007). Porém, durante esse transporte, pode haver problemas de estresse dos peixes.

O estresse ocasionado pelo transporte pode levar à mortalidade de $P$. oligospila. Para compensar os índices de mortalidade na produção, aumentase o número de captura, o que acarreta a elevação dos custos econômicos e ambientais. Assim, conhecer a resposta do peixe aos fatores limitantes de sua cadeia produtiva permite que melhorias sejam implementadas no manejo de transporte, para garantir a sustentabilidade local da atividade. Para tanto, a hematologia pode ser uma ferramenta simples e de baixo custo, com capacidade de oferecer informações importantes sobre o estado geral dos peixes devido ao estresse (Brandão et al., 2006; Ranzani-Paiva et al., 2013; Souza Neves et al., 2014), podendo assim, indicar melhorias nas práticas de manejo para esse setor produtivo. Assim, este estudo avaliou a resposta hematológica de $P$. oligospila ao estresse de transporte.

\section{MATERIAL E MÉTODOS}

Foram capturados 70 espécimes de $P$. oligospila $(21,8 \pm 5,9 \mathrm{~g}$ e $9,2 \pm 0,8 \mathrm{~cm})$ na bacia do Rio Guamá (S 01³4'04.6”; W 4701'50.3”), município de Capitão Poço, nordeste do estado do Pará (Brasil), com auxílio de pescadores locais. Os espécimes foram mantidos em tanques de tela submersos no próprio rio, para redução do estresse de captura. Em seguida, após período de aclimatação de aproximadamente 10 dias, realizaram-se as coletas sanguíneas em $10 P$. oligospila para a determinação dos valores hematológicos basais. As coletas de sangue por punção caudal com o uso de seringas contendo EDTA (10\%) foram conduzidas no próprio rio. No rio, no momento da coleta de sangue, os parâmetros de oxigênio dissolvido $(6,3 \pm 0,8 \mathrm{mg} / \mathrm{L})$, o $\mathrm{pH}(6,4 \pm 0,4)$, a condutividade elétrica $(22,8 \pm 0,2 \mu \mathrm{S} / \mathrm{cm})$ e a temperatura $\left(25 \pm 0,4^{\circ} \mathrm{C}\right)$ foram monitorados e, posteriormente, mantidos nos tanques do laboratório para impedir variações de estresse decorrente da qualidade de água.

Os demais peixes foram, então, separados em grupos de 10, totalizando seis grupos, e cada grupo foi acondicionado em sacos plásticos de 20L, com 6L de água, correspondendo a 1/3 do seu volume; os outros $2 / 3$ foram preenchidos com oxigênio, e simularam-se as condições comumente empregadas pelos pescadores locais. Em seguida, os peixes foram transportados durante três horas, via rodovia, até o Laboratório da UFPA, Bragança. Após o transporte, todos os peixes foram aclimatados em caixas com 300L de água e com sistema de recirculação.

Em zero hora (momento de chegada ao laboratório), seis, 24, 48, 72 e 96 horas após o transporte, 10 peixes foram utilizados para coleta de sangue como descrito anteriormente. As amostras de sangue foram usadas para determinação dos níveis de glicose plasmática, utilizando-se medidor automático (Prestige IQ 50), hematócrito, pelo método de microhematócrito (Goldenfarb et al., 1971), concentração da hemoglobina, pelo método da cianometa-hemoglobina e contagem de eritrócitos totais em câmara de Neubauer. De posse dos dados de eritrócitos, hematócrito e hemoglobina, foram calculados os índices hematimétricos de Witronbe: volume corpuscular médio (VCM), hemoglobina corpuscular média 
(HCM) e concentração de hemoglobina corpuscular média (CHCM). Para a determinação dos níveis de proteína plasmática total, utilizou-se refratômetro (Quimis ${ }^{\circledR}$ ). Extensões sanguíneas foram confeccionadas e coradas pancromicamente com Giemsa e May Grünwald para a contagem de leucócitos e trombócitos totais, bem como para a contagem diferencial de leucócitos (Ranzani-Paiva et al., 2013).

Os dados obtidos foram submetidos previamente ao teste de normalidade (Shapiro Wilk) e homocedasticidade (teste de Bartlett) e, posteriormente, à análise de variância, seguida pelo o teste de Tukey para comparação entre médias (Zar, 2010).

\section{RESULTADOS E DISCUSSÃO}

Durante o transporte dos peixes, não foi observada qualquer mortalidade dos animais, e, durante aclimatação e amostragens sanguíneas, não houve qualquer surgimento de doenças secundárias ou mortalidade de peixes. Similarmente, o transporte não causou mortalidade em alevinos de Salminus brasiliensis em até 12 horas (Adamante et al., 2008) e em Pimelodus maculatus (Braun e Nuñes, 2014). Em contraste, Gomes et al. (2003) registraram lesões no corpo e mortalidade de $11 \%$ dos alevinos de Colossoma macropomum após três horas de transporte. O transporte é um fator estressante que pode diminuir a resistência imunológica dos peixes. Esses se tornam susceptíveis às infeções primárias (parasitárias) e secundárias (bacterianas), as quais podem levar à mortalidade no cultivo (Takahashi et al., 2006; Lemos et al., 2015).

Após o transporte de $P$. oligospila, houve aumento $(\mathrm{P}<0,05)$ nos níveis de glicose plasmática em zero hora quando comparados aos níveis basais, com retorno aos níveis basais em seis horas, mantendo-se estável pelo período deste estudo (Fig. 1). Similarmente, aumento na glicemia após estresse foi relatada em $C$. macropomum (Gomes et al., 2003) e Arapaima gigas (Brandão et al., 2006), mas com diferenças no tempo de retorno aos níveis basais, que podem estar relacionadas à intensidade do estímulo estressor e à espécie de peixe estudada. Em peixes estressados, a hiperglicemia é consequência do aumento das catecolaminas no sangue, que aumentam a glicogenólise no fígado na tentativa de atender a maior demanda energética do organismo (Adamante et al., 2008; Baldisserotto, 2009; Souza Neves et al., 2014).

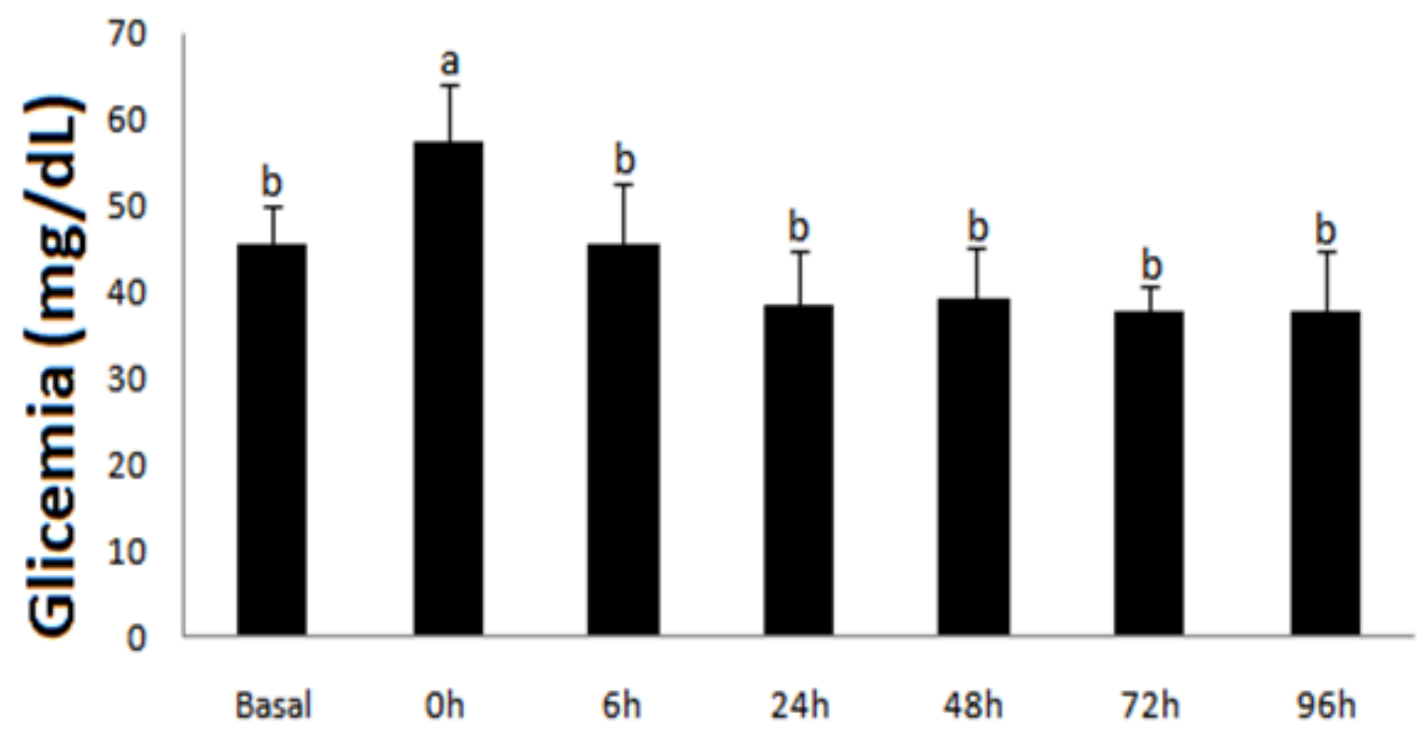

Figura 1. Valores médios \pm desvio-padrão da glicemia plasmática do Peckoltia oligospila antes (basal) e após ser submetido ao estresse de transporte. Valores na mesma coluna com letras iguais não diferem pelo teste de Tukey $(\mathrm{P}>0,05)$. 
Tanto o número de eritroblastos (Fig. 2A) quanto o de eritrócitos totais (Fig. 2B) aumentaram em zero hora do transporte e, posteriormente, reduziram após seis horas de transporte, retornando aos níveis basais em 24 horas. Tais aumentos estão relacionados à elevação das catecolaminas circulantes decorrentes do estresse, o que provoca a contração esplênica e aumenta a quantidade dessas células na circulação, devido à estimulação da eritropoiese (Tavares-Dias e Moraes, 2004; Baldisserotto, 2009).

Os níveis de proteínas plasmáticas totais estão diretamente relacionados com o bom funcionamento do organismo dos animais, pois atuam no transporte de metabólitos, na defesa humoral e na coagulação sanguínea (Satake et al., 2009). Neste estudo, observaram-se aumento nos níveis de proteínas plasmáticas totais (Fig. 2C) em seis horas do transporte dos peixes e redução em $72 \mathrm{~h}$ do transporte. Durante estresse, distúrbios osmorregulatórios podem afetar o
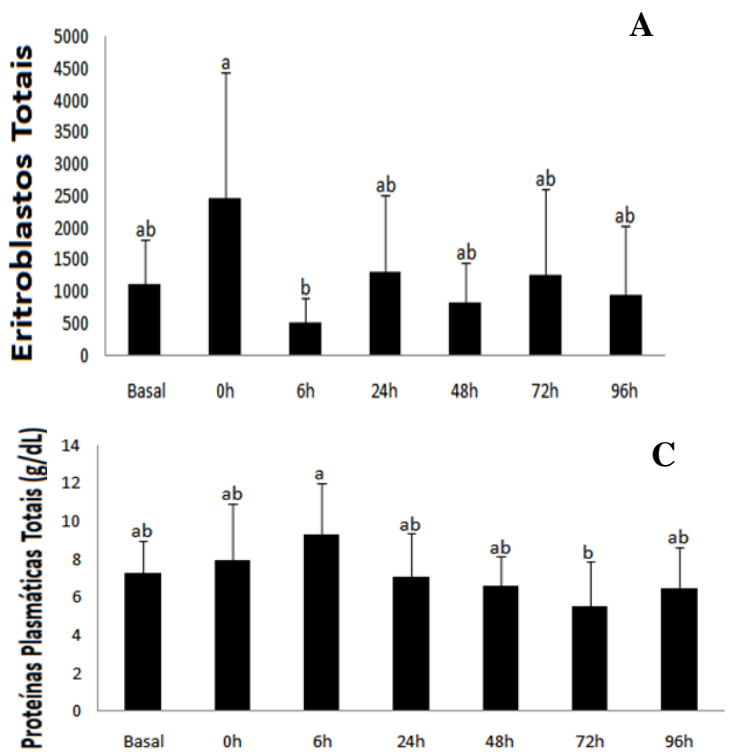

catabolismo de proteínas (Satake et al., 2009), causando essa hipoproteinemia. Assim, os níveis de proteínas totais podem ser indicadores de estresse em peixes (Inoue et al., 2005). Tais distúrbios também levaram a uma diminuição do hematócrito (Fig. 2D) entre 48 e 72 horas do transporte, a qual pode estar relacionada com perda de água do plasma sanguíneo (Urbinati et al., 2004), uma vez que o número de eritrócitos totais e o VCM não se alteram nesses períodos de estresse. Em A. gigas submetido a estresse de transporte, houve redução no hematócrito (Brandão et al., 2006), enquanto em Pseudoplatystoma corruscans houve aumento em 12 e 24 horas após transporte (Fagundes e Urbinati, 2008). Porém, em C. macropomum, nenhuma alteração nos níveis de hematócrito foi relatada (Gomes et al., 2003). Portanto, em $P$. oligospila, essa redução no hematócrito, em 48 e 72 horas, foi uma resposta tardia ao estresse de transporte.
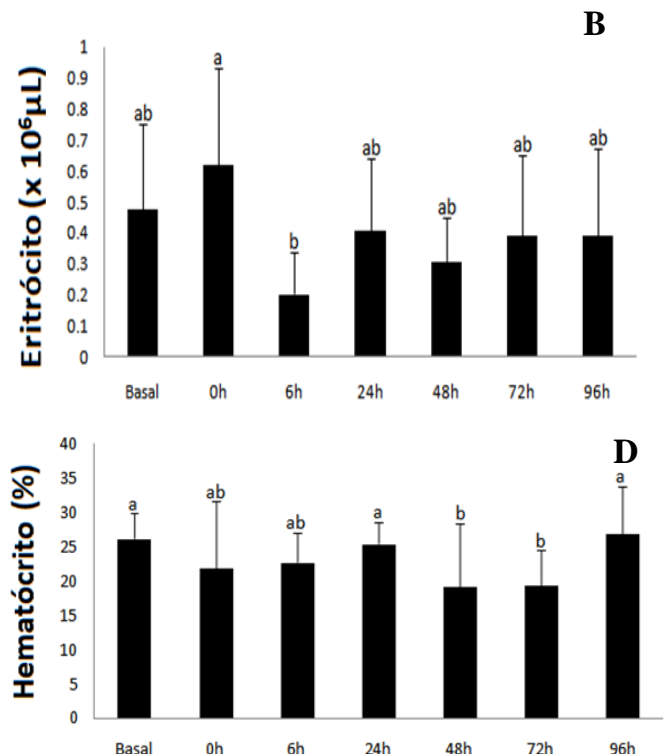

Figura 2. Parâmetros hematológicos do Peckoltia oligospila antes (basal) e após ser submetido ao estresse de transporte. A) Eritroblastos totais; B) eritrócito; C) proteínas plasmáticas totais; D) hematócrito. Valores na mesma coluna com letras iguais não diferem pelo teste de Tukey $(\mathrm{P}>0,05)$.

Em P. oligospila, o HCM (Fig. 3A) e o VCM (Fig. 3B) apresentam aumento em seis horas após transporte, mas o HCM retornou aos valores basais em 24 horas, enquanto o VCM aumentou em 96 horas após transporte. Níveis de hemoglobina e CHCM permaneceram inalterados. Porém, em outros peixes submetidos a diferentes condições de estresse, não houve alteração nos índices hematimétricos (Silva et al., 2012; Fujimoto et al., 2013). 
Os leucócitos observados no sangue de $P$. oligospila foram linfócitos, monócitos e neutrófilos, com características similares a de outros peixes (Tavares-Dias e Moraes, 2004). Além disso, os números de leucócitos e trombócitos não mostraram alterações significativas $(\mathrm{P}>0,05)$ em decorrência do estresse de transporte (Tab. 1). Porém, em

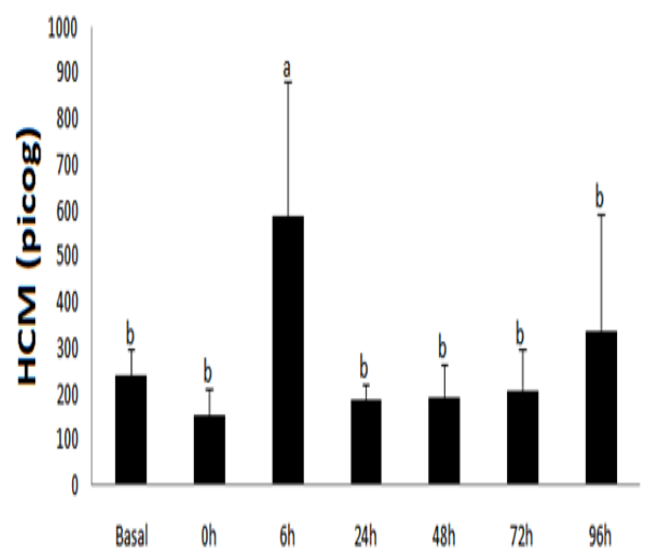

algumas situações de estresse, os peixes podem apresentar linfocitopenia, neutrofilia e monocitofilia (Tavares-Dias e Moraes, 2004; Ranzani-Paiva et al., 2013). Todavia, em $P$. oligospila, a magnitude do estímulo estressor usado não foi suficiente para comprometer o sistema imunológico celular.

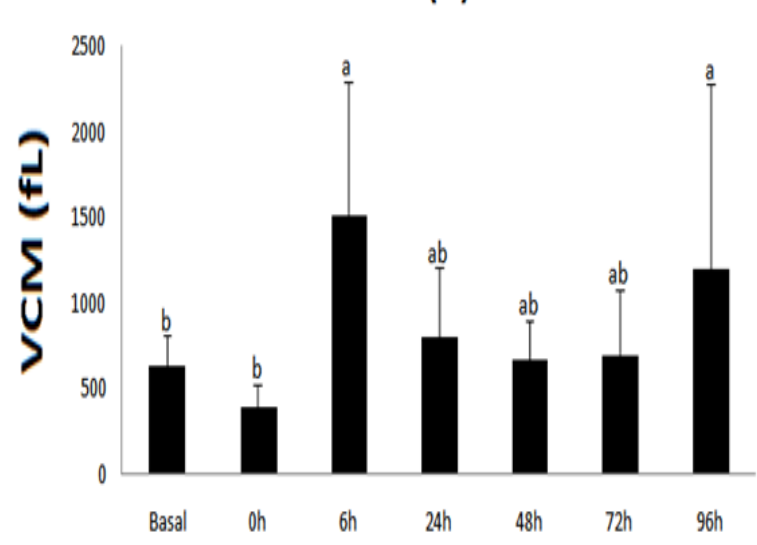

Figura 3. Parâmetros hematológicos do Peckoltia oligospila antes (basal) e após ser submetido ao estresse de transporte. A) HCM e B) VCM. Valores na mesma coluna com letras iguais não diferem pelo teste de Tukey $(\mathrm{P}>0,05)$.

Tabela 1. Parâmetros hematológicos do Peckoltia oligospila antes e após estresse de transporte. Valores expressam média \pm desvio-padrão, $\mathrm{n}=70$

\begin{tabular}{cccccccc}
\hline Parâmetros & Basal & 0 hora & 6 horas & 24 horas & 48 horas & 72 horas & 96 horas \\
\hline Hemoglobina & $9,00 \pm 0,8$ & $8,31 \pm 3,7$ & $8,88 \pm 1,2$ & $8,59 \pm 3,0$ & $6,18 \pm 6,1$ & $6,28 \pm 2,6$ & $8,83 \pm 3,7$ \\
$(\mathrm{~g} / \mathrm{dL})$ & $0 \mathrm{a}$ & $\mathrm{a}$ & $0 \mathrm{a}$ & $\mathrm{a}$ & $\mathrm{a}$ & $\mathrm{a}$ & $0 \mathrm{a}$ \\
$\mathrm{CHCM}(\mathrm{g} / \mathrm{dL})$ & $35,58 \pm 2$, & $39,88 \pm 10$ & $37,99 \pm 4$, & $31,58 \pm 9$, & $29,8 \pm 19$, & $33,61 \pm 12$ & $33,75 \pm 13$ \\
Leucócitos $\left(10^{3} \mathrm{x}\right.$ & $9,36 \pm 3,1$ & $14,24 \pm 7$, & $9,49 \pm 8,3$ & $11,84 \pm 6$, & $7,83 \pm 3,5$ & $10,12 \pm 8$, & $9,97 \pm 7,7$ \\
$\mu \mathrm{L})$ & $0 \mathrm{a}$ & $8 \mathrm{a}$ & $0 \mathrm{a}$ & $2 \mathrm{a}$ & $\mathrm{a}$ & $00 \mathrm{a}$ & $\mathrm{a}$ \\
Trombócitos $\left(10^{3}\right.$ & $3,53 \pm 1,3$ & $3,62 \pm 1,8$ & $1,44 \pm 0,7$ & $2,78 \pm 2,1$ & $2,84 \pm 1,9$ & $3,67 \pm 3,2$ & $3,75 \pm 3,5$ \\
$\mathrm{x} \mu \mathrm{L})$ & $0 \mathrm{a}$ & $\mathrm{a}$ & $0 \mathrm{a}$ & $\mathrm{a}$ & $\mathrm{a}$ & $0 \mathrm{a}$ & $\mathrm{a}$ \\
Linfócitos $\left(10^{3} \mathrm{x}\right.$ & $6,72 \pm 2,3$ & $9,62 \pm 5,6$ & $3,94 \pm 2,5$ & $9,19 \pm 4,6$ & $5,08 \pm 2,3$ & $7,30 \pm 6,3$ & $7,07 \pm 5,9$ \\
$\mu \mathrm{L})$ & $\mathrm{a}$ & $\mathrm{a}$ & $0 \mathrm{a}$ & $\mathrm{a}$ & $\mathrm{a}$ & $0 \mathrm{a}$ & $0 \mathrm{a}$ \\
Neutrófilos $\left(10^{3} \mathrm{x}\right.$ & $2,09 \pm 0,8$ & $3,79 \pm 2,0$ & $3,34 \pm 2,8$ & $2,00 \pm 1,4$ & $2,28 \pm 1,1$ & $2,29 \pm 1,6$ & $2,35 \pm 1,7$ \\
$\mu \mathrm{L})$ & $\mathrm{a}$ & $\mathrm{a}$ & $0 \mathrm{a}$ & $0 \mathrm{a}$ & $\mathrm{a}$ & $0 \mathrm{a}$ & $0 \mathrm{a}$ \\
Monócitos $\left(10^{3} \mathrm{x}\right.$ & $0,62 \pm 0,4$ & $0,84 \pm 0,4$ & $0,52 \pm 0,4$ & $0,65 \pm 0,5$ & $0,47 \pm 0,2$ & $0,48 \pm 0,3$ & $0,54 \pm 0,4$ \\
$\mu \mathrm{L})$ & $1 \mathrm{a}$ & $6 \mathrm{a}$ & $9 \mathrm{a}$ & $2 \mathrm{a}$ & $8 \mathrm{a}$ & $5 \mathrm{a}$ & $6 \mathrm{a}$
\end{tabular}

CHCM: concentração de hemoglobina corpuscular média. A soma de alguns valores de diferencial de leucócitos não corresponde ao valor total de leucócitos, em decorrência da retirada de outliers. Letras iguais na mesma linha não apresentam diferença significativa pelo teste de Tukey $(\mathrm{P}>0,05)$.

\section{CONCLUSÕES}

Nas condições de transporte investigadas, Peckoltia oligospila mostrou resistência ao estresse de transporte. Contudo, é recomendado que não seja realizado qualquer outro procedimento estressante por, no mínimo, 24 horas após transporte, para sua recuperação fisiológica adequada, visando assegurar maior higidez e sobrevivência dos peixes. 


\section{AGRADECIMENTOS}

À Capes, pela Bolsa de Pós-Graduação concedida ao primeiro autor, e ao $\mathrm{CNPq}$, pela Bolsa Produtividade concedida ao Dr. Marcos Tavares Dias e Dr. Rodrigo Yudi Fujimoto.

\section{REFERÊNCIAS}

ADAMANTE, W.B.; NUÑER, A.P.O; BARCELLOS, L.J.G. et al. Stress in Salminus brasiliensis fingerlings due to different densities and times of transportation. Arq. Bras. Med. Vet. Zootec., v.60, p.755-761, 2008.

BALDISSEROTTO, B. Fisiologia de peixes aplicada a piscicultura. 2.ed. Santa Maria: UFSM, 2009. 350p.

BRANDÃO, F.R.; GOMES, L.C.; CHAGAS, E.C. Respostas de estresse em pirarucu (Arapaima gigas) durante práticas de rotina em piscicultura. Acta Amaz., v.3, p.349-356, 2006.

BRAUN, N.; NUÑER, A.P.O. Stress in Pimelodus maculatus (Siluriformes: Pimelodidae) at different densities and times in a simulated transport. Zoologia, v.31, p.101-104, 2014.

CHAO, N.L. Fisheries, diversity, and conservation of ornamental fishes of the Rio Negro Basin, Brazil - a review of project piaba. In: CHAO, N.L.; PETRY, P.; PRANG, G. et al. (Eds.). Conservation and management of ornamental fish resources of the Rio Negro Basin, Amazonia, Brazil. Manaus: Universidade do Amazonas, 2001. p.161-204.

EL-GALIL, M.A.A.; ABOELHADID， S.M. Trials for the control of trichodinosis and gyrodactylosis in hatchery reared Oreochromis niloticus fries by using garlic. Vet. Parasitol., v.185, p.57-63, 2012.

FAGUNDES, M.; URBINATI, E.C. Stress in pintado (Pseudoplatystoma corruscans) during farming procedures. Aquaculture, v.276, p.112119,2008

FUJIMOTO, R.Y.; NEVES, M.S.; SANTOS, R.F.B. et al. Morphological and hematological studies of Trypanosoma spp. infecting ornamental armored catfish from Guamá RiverPA, Brazil. Anais Acad. Bras. Cienc., v.85, p.1149-1156, 2013.
GOLDENFARB, P.B.; BOWYER, F.P.; HALL, E.; BROSIOUS, E. Reproducibility in the hematology laboratory: the microhematocrit determination. Am. J. Clin. Pathol., v.56, p.3539, 1971.

GOMES, L.C.; ARAUJO-LIMA, C.A.R.M.; ROUBACH, R.; URBINAT, E.C. Avaliação dos efeitos do sal e da densidade no transporte de tambaqui. Pesqui. Agropec. Bras., v.38, p.283290, 2003.

GUNTHER, A. Catalogue of the fishes in the British. Museum, v.5. p.1-455, 1864.

HARPER, C. Introducing Ichthyophthirius multifiliis - adevastating parasite. Aquacult. Mag., v.29, p.49-52, 2003.

INOUE, L.A.K.A.; AFONSO, L.O.; IWAMA, G.K.; MORAES, G. Effects of clove oil on the stress response of matrinxã (Brycon cephalus) subjected to transport. Acta Amaz., v.35, p.289295, 2005.

LEMOS, J.R.G.; OLIVEIRA, A.T.; SANTOS, M.Q.C.; PEREIRA, C.N.; NASCIMENTO, R.B.; TAVARES-DIAS, M. Influência do transporte na relação peso-comprimento e fator de condição de Paracheirodon axelrodi (Characidae). Biota Amaz., v.5, p.22-26, 2015.

OLIVEIRA, J.R.; CARMO, J.L.; OLIVEIRA, K.K.C.; SOARES, M.C.F. Cloreto de sódio, benzocaine e oleo de cravo-da-índia na água de transporte de tilápia-do-nilo. Rev. Bras. Zootec., v.38, p.1163-1169, 2009.

PRANG, G. An industry analysis of the freshwater ornamental fishery with particular reference to the supply of Brazilian freshwater ornamentals to the UK market. Uakari, v.3, p.7$51,2007$.

RANZANI-PAIVA, M.J.T.; PADUA, S.B.; TAVARES-DIAS, M.; EGAMI, M.I. Métodos para análises hematológicas em peixes. Maringá: EDUEM, 2013. 144p.

RIBEIRO, F.A.S.; CARVALHO JUNIOR, J.R.; FERNANDES, J.B.K.; NAKAYAMA, L. Cadeia produtiva do peixe ornamental. Panorama Aquicul., v.19, p.36-45, 2009. 
SATAKE, M.M.; HISANO, H.; PÁDUA, S.B.; TAVARES-DIAS, M. Relação pesocomprimento, fator de condição e parâmetros hematológicos de dourado (Salminus brasiliensis) cultivado em condições experimentais. Bol. Pesqui. Des. Embrapa Agropec. Oeste, n.51, p.1-22, 2009.

SILVA, R.D.; ROCHA, L.O.; FORTES, B.D.A.; VIERA, D.; FIORAVANTI, M.C.S. Parâmetros hematológicos e bioquímicos da tilápia-do-Nilo (Oreochromis niloticus L.) sob estresse por exposição ao ar. Pesqui. Vet. Bras., v.32, p.99107,2012

SOUZA NEVES, M.; BARBAS, L.A.L.; FUJIMOTO, R.Y. Hematology and recovery response in jacundá, Crenicichla saxatilis (Linnaeus, 1758) after short-term handling stress. J. Appl. Ichthyol., v.30, p.42-47, 2014.

TAKAHASHI, L.S.; ABREU, J.S.; BILLER, J.D.; URBINATI, E.C. Efeito do ambiente pós-transporte na recuperação dos indicadores de estresse de pacus juvenis, Piaractus mesopotamicus. Acta Sci. Anim. Sci., v.28, p.469-475, 2006.
TAVARES-DIAS M.; MORAES F.R. Hematologia de peixes teleósteos. Ribeirão Preto: Villimpress, 2004. 144p.

TORRES, M.F. A pesca ornamental na bacia do Rio Guamá: sustentabilidade e perspectivas ao manejo. 2007. 264f. Tese (Doutorado) - Núcleo de Altos Estudos Amazônicos, Universidade Federal do Pará, Belém, PA.

URBINATI, E.C.; ABREU, J.S.; CAMARGO, A.C.S.; LANDINES, M.A.P. Loading and transport stress of juvenile matrinxã (Brycon cephalus, Characidae) at various densities. Aquaculture, v.229, p.389-400, 2004.

ZAR, J.H. Biostatistical analysis. 5.ed. New Jersey: Prentice Hall, 2010. 944p. 